\title{
Research Article \\ Fixed Point Theorem for Cyclic Chatterjea Type Contractions
}

\author{
Erdal Karapinar $^{1}$ and Hemant Kumar Nashine ${ }^{2}$ \\ ${ }^{1}$ Department of Mathematics, Attlım University, Incek, 06836 Ankara, Turkey \\ ${ }^{2}$ Department of Mathematics, Disha Institute of Management and Technology, Satya Vihar, \\ Vidhansabha-Chandrakhuri Marg, Mandir Hasaud, Raipur 492101, India \\ Correspondence should be addressed to Erdal Karapınar, erdalkarapinar@yahoo.com
}

Received 23 April 2012; Revised 1 August 2012; Accepted 1 August 2012

Academic Editor: Saeid Abbasbandy

Copyright (C) 2012 E. Karapınar and H. K. Nashine. This is an open access article distributed under the Creative Commons Attribution License, which permits unrestricted use, distribution, and reproduction in any medium, provided the original work is properly cited.

We introduce the notion of cyclic weakly Chatterjea type contraction and generalized cyclic weakly Chatterjea type contraction in metric spaces. We discussed the existence of fixed point theorems of (generalized) cyclic weakly Chatterjea type contraction mappings in the context of complete metric spaces. Our main theorems extend and improve some fixed point theorems in the literature.

\section{Introduction and Preliminaries}

Celebrated Banach's contraction mapping principle is one of the cornerstones in the development of nonlinear analysis. Fixed point theorems have applications not only in the various branches of mathematics but also in economics, chemistry, biology, computer science, engineering, and others. In particular, such theorems are used to demonstrate the existence and uniqueness of a solution of differential equations, integral equations, functional equations, partial differential equations, and others. Due to the importance, generalizations of Banach fixed point theorem have been investigated heavily by many authors. (see, e.g., [1, 2]).

Following this trend, in 1972, Chatterjea [3] introduced the following definition.

Definition 1.1. Let $(X, d)$ be a metric space. A self-mapping $T: X \rightarrow X$ is called a Chatterjea type contraction if there exists $\alpha \in(0,1 / 2)$ such that for all $x, y \in X$ the following inequality holds:

$$
d(T x, T y) \leq \alpha(d(x, T y)+d(y, T x))
$$


In this interesting paper, Chatterjea [3] proved that every Chatterjea type contraction $T$ on a complete metric space has a unique fixed point. Later, Choudhury [4] introduced a generalization of Chatterjea type contraction as follows.

Definition 1.2. A self-mapping $T: X \rightarrow X$, on a metric space $(X, d)$, is said to be a weakly $C$-contractive (or a weak Chatterjea type contraction) if for all $x, y \in X$,

$$
d(T x, T y) \leq \frac{1}{2}[d(x, T y)+d(y, T x)]-\psi(d(x, T y), d(y, T x))
$$

where $\psi:[0,+\infty)^{2} \rightarrow[0,+\infty)$ is a continuous function such that

$$
\psi(x, y)=0 \quad \text { iff } x=y=0 .
$$

In [4], the author proves that every weak Chatterjea type contraction on a complete metric space has a unique fixed point.

One of the interesting generalizations of a Banach's contraction mapping principle was given by Kirk et al. [5] in 2003 by introducing the following notion of cyclic representation.

Definition 1.3 (see [5]). Let $X$ be a nonempty set and let $T: X \rightarrow X$ be an operator. By definition, $X=\bigcup_{i=1}^{m} X_{i}$ is a cyclic representation of $X$ with respect to $T$ if

(a) $X_{i}, i=1, \ldots, m$ are nonempty sets,

(b) $T\left(X_{1}\right) \subset X_{2}, \ldots, T\left(X_{m-1}\right) \subset X_{m}, T\left(X_{m}\right) \subset X_{1}$.

After the remarkable paper of Kirk et al. [5], some new fixed-point theorems for operators $T$ defined on a complete metric space $X$ with a cyclic representation of $X$ with respect to $T$ have appeared in the literature (see, e.g., [1, 2, 4-19]). Very recently, Karapinar [10] introduced the notion of the cyclic weak $\varphi$-contraction and proved fixed point theorems for these types of contractions.

Definition 1.4 (see [10]). Let $(X, d)$ be a metric space, $m \in \mathbb{N}, A_{1}, A_{2}, \ldots, A_{m}$ closed nonempty subsets of $X$, and $Y=\bigcup_{i=1}^{m} A_{i}$. An operator $T: Y \rightarrow Y$ is called a cyclic weak $\varphi$-contraction if

(1) $X=\bigcup_{i=1}^{m} A_{i}$ is a cyclic representation of $X$ with respect to $T$;

(2) there exists a continuous, nondecreasing function $\varphi:[0, \infty) \rightarrow[0, \infty)$ with $\varphi(t)>0$ for $t \in(0, \infty)$ and $\varphi(0)=0$ such that

$$
d(T x, T y) \leq d(x, y)-\varphi(d(x, y))
$$

for any $x \in A_{i}, y \in A_{i+1}, i=1,2, \ldots, m$ where $A_{m+1}=A_{1}$.

Let $\mathbf{F}$ denote all the continuous functions $\varphi:[0, \infty) \rightarrow[0, \infty)$ with $\varphi(t)>0$ for $t \in$ $(0, \infty)$ and $\varphi(0)=0$.

Theorem 1.5 (see [10]). Let $(X, d)$ be a complete metric space, $m \in \mathbb{N}, A_{1}, A_{2}, \ldots, A_{m}$ nonempty subsets of $X$, and $X=\bigcup_{i=1}^{m} A_{i}$. Suppose that $T$ is a cyclic weak $\varphi$-contraction with $\varphi \in \mathbf{F}$. Then, $T$ has a fixed point $z \in \bigcap_{i=1}^{n} A_{i}$. 
In this paper, we introduce the notions of cyclic weakly Chatterjea type contractions and generalized cyclic weakly Chatterjea type contractions and then derive fixed point theorems on these cyclic contractions in the setup of complete metric spaces. Our results generalize fixed point theorems $[2,5,20]$ in the sense of metric spaces.

\section{Main Results}

\subsection{Fixed Point Theory for the Cyclic Weakly Chatterjea Type Contractions in Complete Metric Space}

First we introduce the notion of cyclic weakly Chatterjea type contraction in metric space.

For convenience, we denote by $\mathbf{F}_{1}$ the class of functions $\psi:[0, \infty)^{2} \rightarrow[0, \infty)$ lower semicontinuous satisfying $\psi(x, y)=0$ if and only if $x=y=0$.

Definition 2.1. Let $(X, d)$ be a metric space, $m \in \mathbb{N}, A_{1}, A_{2}, \ldots, A_{m}$ nonempty subsets of $X$, and $Y=\bigcup_{i=1}^{m} A_{i}$. An operator $T: Y \rightarrow Y$ is called a cyclic weakly Chatterjea type contraction if

(1) $X=\bigcup_{i=1}^{m} A_{i}$ is a cyclic representation of $X$ with respect to $T$;

(2) for any $x \in A_{i}, y \in A_{i+1}, i=1,2, \ldots, m$

$$
d(T x, T y) \leq \alpha(d(x, T y)+d(y, T x))-\psi(d(x, T y), d(y, T x))
$$

where $A_{m+1}=A_{1}, \psi \in \mathbf{F}_{1}$ and $\alpha \in(0,1 / 2]$.

Notice that the cyclic weak Chatterjea type contractions constitute a strictly larger class of mappings than cyclic weak $\varphi$-contractions.

The main result of this section is the following.

Theorem 2.2. Let $(X, d)$ be a complete metric space, $m \in \mathbb{N}, A_{1}, A_{2}, \ldots, A_{m}$ nonempty closed subsets of $X$, and $X=\bigcup_{i=1}^{m} A_{i}$. Suppose that $T$ is a cyclic weakly Chatterjea type contraction. Then, $T$ has a unique fixed point $z \in \bigcap_{i=1}^{n} A_{i}$.

Proof. Take $x_{0} \in X$ and consider the sequence given by $x_{n+1}=T x_{n}, n=0,1,2, \ldots$ If there exists $n_{0} \in \mathbb{N}$ such that $x_{n_{0}+1}=x_{n_{0}}$, then the existence of the fixed point is proved. Indeed, $x_{n_{0}+1}=T x_{n_{0}}=x_{n_{0}}$ and $x_{n_{0}}$ is the desired point. Suppose that $x_{n+1} \neq x_{n}$ for all $n=0,1,2, \ldots$ Since $X=\bigcup_{i=1}^{m} A_{i}$, for any $n>0$ there exists $i_{n} \in\{1,2, \ldots, m\}$ such that $x_{n-1} \in A_{i_{n}}$ and $x_{n} \in A_{i_{n+1}}$. Due to the fact that $T$ is a cyclic weakly Chatterjea type contraction, we have

$$
\begin{aligned}
d\left(x_{n}, x_{n+1}\right) & =d\left(T x_{n-1}, T x_{n}\right) \\
& \leq \alpha\left(d\left(x_{n-1}, T x_{n}\right)+d\left(x_{n}, T x_{n-1}\right)\right)-\psi\left(d\left(x_{n-1}, T x_{n}\right), d\left(x_{n}, T x_{n-1}\right)\right) \\
& =\alpha\left(d\left(x_{n-1}, x_{n+1}\right)+d\left(x_{n}, x_{n}\right)\right)-\psi\left(d\left(x_{n-1}, x_{n+1}\right), 0\right) \leq \alpha d\left(x_{n-1}, x_{n+1}\right) \\
& \leq \alpha\left(d\left(x_{n-1}, x_{n}\right)+d\left(x_{n}, x_{n+1}\right)\right) .
\end{aligned}
$$


Therefore,

$$
d\left(x_{n}, x_{n+1}\right) \leq \frac{\alpha}{1-\alpha} d\left(x_{n-1}, x_{n}\right) \text { for any } n=1,2, \ldots
$$

If we take $k=\alpha /(1-\alpha)<1$, then we have

$$
d\left(x_{n}, x_{n+1}\right) \leq k d\left(x_{n-1}, x_{n}\right) \text { for any } n=1,2, \ldots
$$

It follows that $d\left(x_{n}, x_{n+1}\right) \leq k^{n} d\left(x_{0}, x_{1}\right)$ and

$$
\lim _{n \rightarrow \infty} d\left(x_{n}, x_{n+1}\right)=0
$$

In the sequel, we will prove that $\left\{x_{n}\right\}$ is a Cauchy sequence. First, we prove the following claim. For every $\varepsilon>0$ there exists $n \in \mathbb{N}$ such that if $r, q \geq n$ with $r-q \equiv 1(m)$, then $d\left(x_{r}, x_{q}\right)<$ $\varepsilon$.

Suppose, to the contrary, that there exists $\varepsilon>0$ such that for any $n \in \mathbb{N}$ we can find $r_{n}>q_{n} \geq n$ with $r_{n}-q_{n} \equiv 1(m)$ satisfying

$$
d\left(x_{q_{n}}, x_{r_{n}}\right) \geq \varepsilon
$$

Now, we take $n>2 m$. Then, corresponding to $q_{n} \geq n$ we can choose $r_{n}$ in such a way that it is the smallest integer with $r_{n}>q_{n}$ satisfying $r_{n}-q_{n} \equiv 1(m)$ and $d\left(x_{q_{n}}, x_{r_{n}}\right) \geq \varepsilon$. Therefore, $d\left(x_{q_{n}}, x_{r_{n-m}}\right) \leq \varepsilon$. Using the triangular inequality, we obtain

$$
\begin{aligned}
\varepsilon \leq d\left(x_{q_{n}}, x_{r_{n}}\right) & \leq d\left(x_{q_{n}}, x_{r_{n-m}}\right)+\sum_{i=1}^{m} d\left(x_{r_{n-i}}, x_{r_{n-i+1}}\right) \\
& <\varepsilon+\sum_{i=1}^{m} d\left(x_{r_{n-i}}, x_{r_{n-i+1}}\right) .
\end{aligned}
$$

Taking (2.5) into account and letting $n \rightarrow \infty$ in the inequality above, we find

$$
\lim _{n \rightarrow \infty} d\left(x_{q_{n}}, x_{r_{n}}\right)=\varepsilon
$$

Again, by the triangular inequality, we have

$$
\begin{aligned}
\varepsilon & \leq d\left(x_{q_{n}}, x_{r_{n}}\right) \\
& \leq d\left(x_{q_{n}}, x_{q_{n+1}}\right)+d\left(x_{q_{n+1}}, x_{r_{n+1}}\right)+d\left(x_{r_{n+1}}, x_{r_{n}}\right) \\
& \leq d\left(x_{q_{n}}, x_{q_{n+1}}\right)+d\left(x_{q_{n+1}}, x_{q_{n}}\right)+d\left(x_{q_{n}}, x_{r_{n}}\right)+d\left(x_{r_{n}}, x_{r_{n+1}}\right)+d\left(x_{r_{n+1}}, x_{r_{n}}\right) \\
& =2 d\left(x_{q_{n}}, x_{q_{n+1}}\right)+d\left(x_{q_{n}}, x_{r_{n}}\right)+2 d\left(x_{r_{n}}, x_{r_{n+1}}\right) .
\end{aligned}
$$


Passing to the limit as $n \rightarrow \infty$ in (2.8) and taking (2.5) and (2.8) into account, we get

$$
\lim _{n \rightarrow \infty} d\left(x_{q_{n+1}}, x_{r_{n+1}}\right)=\varepsilon
$$

Since $x_{q_{n}}$ and $x_{r_{n}}$ lie in different adjacently labeled sets $A_{i}$ and $A_{i+1}$ for certain $1 \leq i \leq m$, using the fact that $T$ is a cyclic weakly Chatterjea type contraction, we have

$$
\begin{aligned}
d\left(x_{q_{n+1}}, x_{r_{n+1}}\right)= & d\left(T x_{q_{n}}, T x_{r_{n}}\right) \\
\leq & \alpha\left(d\left(x_{q_{n}}, T x_{r_{n}}\right)+d\left(x_{r_{n}}, T x_{q_{n}}\right)\right)-\psi\left(d\left(x_{q_{n}}, T x_{r_{n}}\right), d\left(x_{r_{n}}, T x_{q_{n}}\right)\right) \\
\leq & \alpha\left(d\left(x_{q_{n}}, x_{r_{n}+1}\right)+d\left(x_{r_{n}}, x_{q_{n}+1}\right)\right)-\psi\left(d\left(x_{q_{n}}, x_{r_{n}+1}\right), d\left(x_{r_{n}}, x_{q_{n}+1}\right)\right) \\
\leq & \alpha\left(d\left(x_{q_{n}}, x_{r_{n}}\right)+d\left(x_{r_{n}}, x_{r_{n}+1}\right)+d\left(x_{r_{n}}, x_{q_{n}}\right)+d\left(x_{q_{n}}, x_{q_{n}+1}\right)\right) \\
& -\psi\left(d\left(x_{q_{n}}, x_{r_{n}+1}\right), d\left(x_{r_{n}}, x_{q_{n}+1}\right)\right) .
\end{aligned}
$$

Taking (2.5) and (2.8) into account together with the lower semicontinuity of $\psi$, the inequalities above yield

$$
\begin{aligned}
\varepsilon & \leq \alpha \cdot \varepsilon-\liminf _{n \rightarrow+\infty} \psi\left(d\left(x_{q_{n}}, x_{r_{n}+1}\right), d\left(x_{r_{n}}, x_{q_{n}+1}\right)\right) \\
& \leq \alpha \cdot \varepsilon-\psi(\varepsilon, \varepsilon) \leq \alpha \cdot \varepsilon
\end{aligned}
$$

as $n \rightarrow \infty$. Hence, $\psi(\varepsilon, \varepsilon)=0$. From the fact that $\psi(x, y)=0 \Leftrightarrow x=y=0$, we have $\varepsilon=0$, which is a contradiction. Therefore, our claim is proved.

We prove that $(X, d)$ is a Cauchy sequence. Fix $\varepsilon>0$. By the claim, we find $n_{0} \in \mathbb{N}$ such that if $r, q \geq n_{0}$ with $r-q \equiv 1(m)$,

$$
d\left(x_{r}, x_{q}\right) \leq \frac{\varepsilon}{2}
$$

Since $\lim _{n \rightarrow \infty} d\left(x_{n}, x_{n+1}\right)=0$, there exists $n_{1} \in \mathbb{N}$ such that

$$
d\left(x_{n}, x_{n+1}\right) \leq \frac{\varepsilon}{2 m}
$$

for any $n \geq n_{1}$. Suppose that $r, s \geq \max \left\{n_{0}, n_{1}\right\}$ with $s>r$. Then, there exists $k \in\{1,2, \ldots, m\}$ such that $s-r \equiv k(m)$. Therefore, we have $s-r+\varphi \equiv 1(m)$ for $\varphi=m-k+1$. So, we derive

$$
d\left(x_{r}, x_{s}\right) \leq d\left(x_{r}, x_{s+j}\right)+d\left(x_{s+j}, x_{s+j-1}\right)+\cdots+d\left(x_{s+1}, x_{s}\right)
$$

By (2.13) and (2.14) and the inequality above, we get

$$
d\left(x_{r}, x_{s}\right) \leq \frac{\varepsilon}{2}+j \times \frac{\varepsilon}{2 m} \leq \frac{\varepsilon}{2}+m \times \frac{\varepsilon}{2 m}=\varepsilon
$$


This proves that $\left(x_{n}\right)$ is a Cauchy sequence. Since $X$ is a complete metric space, there exists $x \in X$ such that $\lim _{n \rightarrow \infty} x_{n}=x$.

Now, we prove that $x$ is a fixed point of $T$. Since $\lim _{n \rightarrow \infty} x_{n}=x$ and, as $X=\bigcup_{i=1}^{m} A_{i}$ is a cyclic representation of $X$ with respect to $T$, the sequence $\left(x_{n}\right)$ has infinite terms in each $A_{i}$ for $i \in\{1,2, \ldots, m\}$. Suppose that $x \in A_{i}, T x \in A_{i+1}$ and we take a subsequence $x_{n_{k}}$ of $\left(x_{n}\right)$ with $x_{n_{k}} \in A_{i-1}$ (the existence of this subsequence is guaranteed by the previously mentioned comment). By using the contractive condition, we obtain

$$
\begin{aligned}
d\left(x_{n_{k+1}}, T x\right) & =d\left(T x_{n_{k}}, T x\right) \\
& \leq \alpha\left(d\left(x_{n_{k}}, T x\right)+d\left(x, T x_{n_{k}}\right)\right)-\psi\left(d\left(x_{n_{k}}, T x\right), d\left(x, T x_{n_{k}}\right)\right) \\
& =\alpha\left(d\left(x_{n_{k}}, T x\right)+d\left(x, x_{n_{k}+1}\right)\right)-\psi\left(d\left(x_{n_{k}}, T x\right), d\left(x, x_{n_{k}+1}\right)\right) .
\end{aligned}
$$

Letting $n \rightarrow \infty$ and by using $x_{n_{k}} \rightarrow x$ together with the lower semicontinuity of $\psi$, we have

$$
\begin{aligned}
d(x, T x) & \leq \alpha d(x, T x)-\liminf _{n \rightarrow+\infty} \psi\left(d\left(x_{n_{k}}, T x\right), d\left(x, x_{n_{k}+1}\right)\right) \\
& \leq \alpha d(x, T x)-\psi(d(x, T x), 0) \leq \alpha d(x, T x)
\end{aligned}
$$

which is a contradiction (since $\alpha<1 / 2)$ unless $d(x, T x)=0$. Therefore, $x$ is a fixed point of $T$.

Finally, to prove the uniqueness of the fixed point, we have $y, z \in X$ with $y$ and $z$ being fixed points of $T$. The cyclic character of $T$ and the fact that $y, z \in X$ are fixed points of $T$ imply that $y, z \in \bigcap_{i=1}^{m} A_{i}$. Using the contractive condition we obtain

$$
d(y, z)=d(T y, T z) \leq \alpha(d(y, T z)+d(z, T y))-\psi(d(y, T z), d(z, T y))
$$

That is,

$$
d(y, z) \leq 2 \alpha d(y, z)-\psi(d(y, z), d(z, y)) \leq 2 \alpha d(y, z)
$$

This gives us $\psi(d(y, z), d(z, y))=0$, and, by our assumption about $\psi, d(y, z)=0$, that is, $y=z$. This finishes the proof.

Corollary 2.3. Let $(X, d)$ be a complete metric space, $m \in \mathbb{N}, A_{1}, A_{2}, \ldots, A_{m}$ nonempty subsets of $X$, and $\mathrm{X}=\bigcup_{i=1}^{m} A_{i}$. Suppose that $T: X \rightarrow X$ be an operator such that

(1) $X=\bigcup_{i=1}^{m} A_{i}$ is a cyclic representation of $X$ with respect to $T$;

(2) there exists $\beta \in[0,1 / 2)$ such that

$$
d(T x, T y) \leq \beta(d(x, T y)+d(y, T x))
$$

for any $x \in A_{i}, y \in A_{i+1}, i=1,2, \ldots, m$ where $A_{m+1}=A_{1}$. Then, $T$ has a fixed point $z \in \bigcap_{i=1}^{n} A_{i}$. 
Proof. Let $\beta \in[0,1 / 2)$. Here, it suffices to take the function $\psi:[0,+\infty)^{2} \rightarrow[0,+\infty)$ as $\psi(a, b)=$ $(\alpha-\beta)(a+b)$. It is clear that $\psi$ satisfies the following conditions:

(1) $\psi(a, b)=0$ if and only if $a=b=0$,

(2) $\psi(x, y)=(\alpha-\beta)(x+y)=\psi(x+y, 0)$.

Hence, we apply Theorems 2.2 for $\alpha \in[1 / 4,1 / 2)$ and get the desired result.

The following corollary gives us a fixed point theorem with a contractive condition of integral type for cyclic contractions.

Corollary 2.4. Let $(X, d)$ be a complete metric space, $m \in \mathbb{N}, A_{1}, A_{2}, \ldots, A_{m}$ nonempty closed subsets of $X$, and $X=\bigcup_{i=1}^{m} A_{i}$. Suppose that $T: X \rightarrow X$ be an operator such that

(i) $X=\bigcup_{i=1}^{m} A_{i}$ is a cyclic representation of $X$ with respect to $T$;

(ii) there exists $\beta \in[0,1 / 2)$ such that

$$
\int_{0}^{d(T x, T y)} \rho(t) d t \leq \beta \int_{0}^{d(x, T y)+d(y, T x)} \rho(t) d t
$$

for any $x \in A_{i}, y \in A_{i+1}, i=1,2, \ldots, m$ where $A_{m+1}=A_{1}$ and $\rho:[0, \infty) \rightarrow[0, \infty)$ is a Lebesgueintegrable mapping satisfying $\int_{0}^{\varepsilon} \rho(t) d t$ for $\varepsilon>0$. Then $T$ has unique fixed point $z \in \bigcap_{i=1}^{m} A_{i}$.

Proof. It is easily proved that the function $\varphi:[0, \infty) \rightarrow[0, \infty)$ given by $\varphi(t)=\int_{0}^{t} \rho(s) d s$ satisfies that $\varphi \in \mathbf{F}_{1}$. Therefore, Corollary 2.3 is obtained from Theorem 2.2, taking as $\varphi$ the previously defined function and as $\psi$ the function $\psi(x, y)=(\alpha-\beta)(x+y)=\varphi(x+y, 0)$.

If in Corollary 2.4, we take $A_{i}=X$ for $i=1,2, \ldots, m$, we obtain the following result.

Corollary 2.5. Let $(X, d)$ be a complete metric space and let $T: X \rightarrow X$ be a mapping such that for any $x, y \in X$,

$$
\int_{0}^{d(T x, T y)} \rho(t) d t \leq \beta \int_{0}^{d(x, T y)+d(y, T x)} \rho(t) d t
$$

where $\rho:[0, \infty) \rightarrow[0, \infty)$ is a Lebesgue-integrable mapping satisfying $\int_{0}^{\varepsilon} \rho(t) d t$ for $\varepsilon>0$ and the constant $\beta \in[0,1 / 2)$. Then $T$ has unique fixed point.

If in Theorem 2.2 we put $A_{i}=X$ for $i=1,2, \ldots, m$ we get the main result of [4].

Corollary 2.6. Let $(X, d)$ be a complete metric space and $T: X \rightarrow X$ a mapping such that for any $x, y \in X$,

$$
d(T x, T y) \leq \alpha(d(x, T y)+d(y, T x))-\psi(d(x, T y)+d(y, T x)),
$$

where $\psi \in \mathbf{F}_{1}$ and $\alpha \in(0,1 / 2)$. Then $T$ has unique fixed point. 
Example 2.7. Let $X=\mathbb{R}$ with the usual metric. Suppose $A_{1}=[0,1], A_{2}=[0,1 / 2]$, and $Y=$ $\bigcup_{i=1}^{2} A_{i}$. Define $T: Y \rightarrow Y$ such that $T x=x / 9$ for all $x \in Y$. It is clear that $\bigcup_{i=1}^{2} A_{i}$ is a cyclic representation of $Y$ with respect to $T$. Furthermore, if $\psi:[0,+\infty)^{2} \rightarrow[0,+\infty)$ is defined by $\psi(x, y)=(x+y) / 9$, then $\psi \in \mathbf{F}_{2}$. Here $T$ is a generalized cyclic weakly Chatterjea type contraction for $\alpha=1 / 2$. To see this fact we examine three cases.

(i) Suppose that $x \geq y$. Then, the inequality (2.1) turns into

$$
\begin{aligned}
d(T x, T y) & =\left|\frac{x}{7}-\frac{y}{7}\right|=\frac{x-y}{7} \leq \frac{1}{2}[d(x, T y)+d(y, T x)]-\psi(d(x, T y), d(y, T x)) \\
& =\frac{1}{2}\left[\left|x-\frac{y}{7}\right|+\left|y-\frac{x}{7}\right|\right]-\frac{1}{9}\left[\left|x-\frac{y}{7}\right|+\left|y-\frac{x}{7}\right|\right] \\
& =\frac{1}{2}\left[x-\frac{y}{7}+\left|y-\frac{x}{7}\right|\right]-\frac{1}{9}\left[x-\frac{y}{7}+\left|y-\frac{x}{7}\right|\right] \\
& =\frac{7}{18}\left[x-\frac{y}{7}+\left|y-\frac{x}{7}\right|\right] .
\end{aligned}
$$

If $y<x / 7$, then (2.25) becomes

$$
\frac{x-y}{7} \leq \frac{7}{18}\left[x-\frac{y}{7}+\frac{x}{7}-y\right]=\frac{4}{9}(x-y)
$$

Hence, (2.1) is satisfied. If $y \geq x / 7$, then (2.25) becomes

$$
\frac{x-y}{7} \leq \frac{7}{18}\left[x-\frac{y}{7}+y-\frac{x}{7}\right]=\frac{1}{3}(x+y)
$$

Thus, (2.1) is true.

(ii) Suppose that $y / 7 \leq x \leq y$. Then, the inequality (2.1) yields that

$$
\begin{aligned}
d(T x, T y) & =\left|\frac{x}{7}-\frac{y}{7}\right|=\frac{y-x}{7} \leq \frac{1}{2}[d(x, T y)+d(y, T x)]-\psi(d(x, T y), d(y, T x)) \\
& =\frac{1}{2}\left[\left|x-\frac{y}{7}\right|+\left|y-\frac{x}{7}\right|\right]-\frac{1}{9}\left[\left|x-\frac{y}{7}\right|+\left|y-\frac{x}{7}\right|\right] \\
& =\frac{7}{18}\left[\left|x-\frac{y}{7}\right|+\left|y-\frac{x}{7}\right|\right] \\
& =\frac{7}{18}\left[x-\frac{y}{7}+y-\frac{x}{7}\right]=\frac{1}{3}(x+y) .
\end{aligned}
$$

So, (2.1) holds. 
(iii) Finally, suppose that $y / 7 \geq x$. Then, the inequality (2.1) yields that

$$
\begin{aligned}
d(T x, T y) & =\left|\frac{x}{7}-\frac{y}{7}\right|=\frac{y-x}{7} \leq \frac{1}{2}[d(x, T y)+d(y, T x)]-\psi(d(x, T y), d(y, T x)) \\
& =\frac{1}{2}\left[\left|x-\frac{y}{7}\right|+\left|y-\frac{x}{7}\right|\right]-\frac{1}{9}\left[\left|x-\frac{y}{7}\right|+\left|y-\frac{x}{7}\right|\right] \\
& =\frac{7}{18}\left[\left|x-\frac{y}{7}\right|+\left|y-\frac{x}{7}\right|\right] \\
& =\frac{7}{18}\left[x-\frac{y}{7}+y-\frac{x}{7}\right]=\frac{1}{3}(y+x) .
\end{aligned}
$$

Hence, (2.1) is true.

Therefore, all conditions of Theorem 1.5 are satisfied, and so $T$ has a fixed point (which is $z=0 \in \bigcap_{i=1}^{2} A_{i}$.)

\subsection{Fixed Point Theory for the Generalized Cyclic Weakly Chatterjea Type Contractions in Complete Metric Space}

In this section we derive fixed point theorems for self-maps satisfying certain generalized cyclic weakly Chatterjea type contractions in a complete metric space.

For convenience, we denote by $\mathbf{F}_{2}$ the class of functions $\psi:[0, \infty)^{4} \rightarrow[0, \infty)$ lower semicontinuous satisfying $\psi(x, y, z, t)=0$ if and only if $x=y=z=t=0$.

We introduce the notion of generalized cyclic weakly Chatterjea type contraction in metric space.

Definition 2.8. Let $(X, d)$ be a metric space, $m \in \mathbb{N}, A_{1}, A_{2}, \ldots, A_{m}$ nonempty subsets of $X$ and $Y=\bigcup_{i=1}^{m} A_{i}$. An operator $T: Y \rightarrow Y$ is called a generalized cyclic weakly Chatterjea type contraction if

(1) $X=\bigcup_{i=1}^{m} A_{i}$ is a cyclic representation of $X$ with respect to $T$;

(2)

$$
\begin{array}{r}
d(T x, T y) \leq \alpha[d(x, T x)+d(y, T y)+d(x, T y)+d(y, T x)] \\
-\psi(d(x, T x), d(x, T y), d(x, T y), d(y, T x))
\end{array}
$$

for any $x \in A_{i}, y \in A_{i+1}, i=1,2, \ldots, m$ where $A_{m+1}=A_{1}$ and

$$
\psi(d(x, T x), d(y, T y), d(x, T y), d(y, T x)) \in \mathbf{F}_{2} \text { and } \alpha \in\left(0, \frac{1}{4}\right] .
$$

We state the main result of this section as follows.

Theorem 2.9. Let $(X, d)$ be a complete metric space, $m \in \mathbb{N}, A_{1}, A_{2}, \ldots, A_{m}$ nonempty closed subsets of $X$ and $X=\bigcup_{i=1}^{m} A_{i}$. Suppose that $T$ is a generalized cyclic weakly C-contraction. Then, $T$ has a unique fixed point $z \in \bigcap_{i=1}^{n} A_{i}$. 
Proof. As in the proof of Theorem 2.2, we take $x_{0} \in X$ and construct a sequence by defining $x_{n+1}=T x_{n}, n=0,1,2, \ldots$ Suppose that there exists $n_{0} \in \mathbb{N}$ such that $x_{n_{0}+1}=x_{n_{0}}$. Then, since $x_{n_{0}+1}=T x_{n_{0}}=x_{n_{0}}$, the existence of the fixed point is proved. So, we assume that $x_{n+1} \neq x_{n}$ for any $n=0,1,2, \ldots$. Since $X=\bigcup_{i=1}^{m} A_{i}$, for any $n>0$, there exists $i_{n} \in\{1,2, \ldots, m\}$ such that $x_{n-1} \in A_{i_{n}}$ and $x_{n} \in A_{i_{n+1}}$. Regarding that $T$ is a generalized cyclic weakly Chatterjea type contraction, we have

$$
\begin{aligned}
d\left(x_{n}, x_{n+1}\right)= & d\left(T x_{n-1}, T x_{n}\right) \\
\leq & \alpha\left[d\left(x_{n-1}, T x_{n-1}\right)+d\left(x_{n}, T x_{n}\right)+d\left(x_{n-1}, T x_{n}\right)+d\left(x_{n}, T x_{n-1}\right)\right] \\
& -\psi\left(d\left(x_{n-1}, T x_{n-1}\right), d\left(x_{n}, T x_{n}\right), d\left(x_{n-1}, T x_{n}\right), d\left(x_{n}, T x_{n-1}\right)\right) \\
= & \alpha\left[d\left(x_{n-1}, x_{n}\right)+d\left(x_{n}, x_{n+1}\right)+d\left(x_{n-1}, x_{n+1}\right)\right] \\
& -\psi\left(d\left(x_{n-1}, x_{n}\right), d\left(x_{n}, x_{n+1}\right), d\left(x_{n-1}, x_{n+1}\right), 0\right) \\
\leq & \alpha\left[d\left(x_{n-1}, x_{n}\right)+d\left(x_{n}, x_{n+1}\right)+d\left(x_{n-1}, x_{n+1}\right)\right] \\
\leq & 2 \alpha\left[d\left(x_{n-1}, x_{n}\right)+d\left(x_{n}, x_{n+1}\right)\right] .
\end{aligned}
$$

Therefore,

$$
d\left(x_{n}, x_{n+1}\right) \leq \frac{2 \alpha}{1-2 \alpha} d\left(x_{n-1}, x_{n}\right) \text { for any } n=1,2, \ldots
$$

If we take $k=\alpha /(1-\alpha)<1$, then we have

$$
d\left(x_{n}, x_{n+1}\right) \leq k d\left(x_{n-1}, x_{n}\right) \text { for any } n=1,2, \ldots
$$

It follows that $d\left(x_{n}, x_{n+1}\right) \leq k^{n} d\left(x_{0}, x_{1}\right)$ and

$$
\lim _{n \rightarrow \infty} d\left(x_{n}, x_{n+1}\right)=0
$$

We show that $\left\{x_{n}\right\}$ is a Cauchy sequence. For this purpose, first, we prove the following claim. For every $\varepsilon>0$ there exists $n \in \mathbb{N}$ such that if $r, q \geq n$ with $r-q \equiv 1(m)$, then $d\left(x_{r}, x_{q}\right)<\varepsilon$.

Assume the contrary. So there exists $\varepsilon>0$ such that for any $n \in \mathbf{N}$ we can find $r_{n}>$ $q_{n} \geq n$ with $r_{n}-q_{n} \equiv 1(m)$ satisfying

$$
d\left(x_{q_{n}}, x_{r_{n}}\right) \geq \varepsilon
$$


Now, we take $n>2 m$. Then, corresponding to $q_{n} \geq n$ we can choose $r_{n}$ in such a way that it is the smallest integer with $r_{n}>q_{n}$ satisfying $r_{n}-q_{n} \equiv 1(m)$ and $d\left(x_{q_{n}}, x_{r_{n}}\right) \geq \varepsilon$. Therefore, $d\left(x_{q_{n}}, x_{r_{n-m}}\right) \leq \varepsilon$ by using the triangular inequality

$$
\begin{aligned}
\varepsilon & \leq d\left(x_{q_{n}}, x_{r_{n}}\right) \leq d\left(x_{q_{n}}, x_{r_{n-m}}\right)+\sum_{i=1}^{m} d\left(x_{r_{n-i}}, x_{r_{n-i+1}}\right) \\
& <\varepsilon+\sum_{i=1}^{m} d\left(x_{r_{n-i}}, x_{r_{n-i+1}}\right) .
\end{aligned}
$$

Passing to the limit as $n \rightarrow \infty$ in the last inequality and taking into account that $\lim _{n \rightarrow \infty} d\left(x_{n}, x_{n+1}\right)=0$, we obtain

$$
\lim _{n \rightarrow \infty} d\left(x_{q_{n}}, x_{r_{n}}\right)=\varepsilon
$$

Again, by the triangular inequality

$$
\begin{aligned}
\varepsilon & \leq d\left(x_{q_{n}}, x_{r_{n}}\right) \\
& \leq d\left(x_{q_{n}}, x_{q_{n+1}}\right)+d\left(x_{q_{n+1}}, x_{r_{n+1}}\right)+d\left(x_{r_{n+1}}, x_{r_{n}}\right) \\
& \leq d\left(x_{q_{n}}, x_{q_{n+1}}\right)+d\left(x_{q_{n+1}}, x_{q_{n}}\right)+d\left(x_{q_{n}}, x_{r_{n}}\right)+d\left(x_{r_{n}}, x_{r_{n+1}}\right)+d\left(x_{r_{n+1}}, x_{r_{n}}\right) \\
& =2 d\left(x_{q_{n}}, x_{q_{n+1}}\right)+d\left(x_{q_{n}}, x_{r_{n}}\right)+2 d\left(x_{r_{n}}, x_{r_{n+1}}\right) .
\end{aligned}
$$

Taking (2.35) and (2.38) into account, we get

$$
\lim _{n \rightarrow \infty} d\left(x_{q_{n+1}}, x_{r_{n+1}}\right)=\varepsilon
$$

as $n \rightarrow \infty$ in (2.38).

Since $x_{q_{n}}$ and $x_{r_{n}}$ lie in different adjacently labeled sets $A_{i}$ and $A_{i+1}$ for certain $1 \leq i \leq m$, using the fact that $T$ is a generalized cyclic weakly Chatterjea type contraction, we have

$$
\begin{aligned}
d\left(x_{q_{n+1}}, x_{r_{n+1}}\right)= & d\left(T x_{q_{n}}, T x_{r_{n}}\right) \\
\leq & \alpha\left[d\left(x_{q_{n}}, T x_{q_{n}}\right)+d\left(x_{r_{n}}, T x_{r_{n}}\right)+d\left(x_{q_{n}}, T x_{r_{n}}\right)+d\left(x_{r_{n}}, T x_{q_{n}}\right)\right] \\
& -\psi\left(d\left(x_{q_{n}}, T x_{q_{n}}\right), d\left(x_{r_{n}}, T x_{r_{n}}\right), d\left(x_{q_{n}}, T x_{r_{n}}\right), d\left(x_{r_{n}}, T x_{q_{n}}\right)\right) \\
\leq & \alpha\left[d\left(x_{q_{n}}, x_{q_{n+1}}\right)+d\left(x_{r_{n}}, x_{r_{n+1}}\right)+d\left(x_{q_{n}}, x_{r_{n+1}}\right)+d\left(x_{r_{n}}, x_{q_{n+1}}\right)\right] \\
& -\psi\left(d\left(x_{q_{n}}, x_{q_{n+1}}\right), d\left(x_{r_{n}}, x_{r_{n+1}}\right), d\left(x_{q_{n}}, x_{r_{n+1}}\right), d\left(x_{r_{n}}, x_{q_{n+1}}\right)\right) .
\end{aligned}
$$


Taking into account (2.35) and (2.38) and the lower semicontinuity of $\psi$, letting $n \rightarrow \infty$ in the last inequality, we obtain

$$
\begin{aligned}
\varepsilon & \leq \alpha[\varepsilon+0+\varepsilon+0]-\liminf _{n \rightarrow+\infty} \psi\left(d\left(x_{q_{n}}, x_{q_{n+1}}\right), d\left(x_{r_{n}}, x_{r_{n+1}}\right), d\left(x_{q_{n}}, x_{r_{n+1}}\right), d\left(x_{r_{n}}, x_{q_{n+1}}\right)\right) \\
& \leq \alpha[\varepsilon+0+\varepsilon+0]-\psi(\varepsilon, 0, \varepsilon, 0) \leq 2 \alpha \cdot \varepsilon
\end{aligned}
$$

and from the last inequality, $\psi(\varepsilon, 0, \varepsilon, 0) \leq-2 \alpha \varepsilon \leq 0$. Therefore $\varphi(\varepsilon, 0, \varepsilon, 0)=0$. From the fact that $\psi(x, y, z, t)=0 \Leftrightarrow x=y=z=t=0$, we have $\varepsilon=0$, which is a contradiction. Therefore, our claim is proved.

In the sequel, we will prove that $(X, d)$ is a Cauchy sequence. Fix $\varepsilon>0$. By the claim, we find $n_{0} \in \mathbb{N}$ such that if $r, q \geq n_{0}$ with $r-q \equiv 1(m)$

$$
d\left(x_{r}, x_{q}\right) \leq \frac{\varepsilon}{2}
$$

Since $\lim _{n \rightarrow \infty} d\left(x_{n}, x_{n+1}\right)=0$, we also find $n_{1} \in \mathbb{N}$ such that

$$
d\left(x_{n}, x_{n+1}\right) \leq \frac{\varepsilon}{2 m}
$$

for any $n \geq n_{1}$. Suppose that $r, s \geq \max \left\{n_{0}, n_{1}\right\}$ and $s>r$. Then there exists $k \in\{1,2, \ldots, m\}$ such that $s-r \equiv k(m)$. Therefore, $s-r+\varphi \equiv 1(m)$ for $\varphi=m-k+1$. So, we have

$$
d\left(x_{r}, x_{s}\right) \leq d\left(x_{r}, x_{s+j}\right)+d\left(x_{s+j}, x_{s+j-1}\right)+\cdots+d\left(x_{s+1}, x_{s}\right)
$$

By (2.43) and (2.44) and from the last inequality, we get

$$
d\left(x_{r}, x_{s}\right) \leq \frac{\varepsilon}{2}+j \times \frac{\varepsilon}{2 m} \leq \frac{\varepsilon}{2}+m \times \frac{\varepsilon}{2 m}=\varepsilon .
$$

This proves that $\left(x_{n}\right)$ is a Cauchy sequence. Since $X$ is a complete metric space, there exists $x \in X$ such that $\lim _{n \rightarrow \infty} x_{n}=x$. In what follows, we prove that $x$ is a fixed point of $T$. In fact, since $\lim _{n \rightarrow \infty} x_{n}=x$ and as $X=\bigcup_{i=1}^{m} A_{i}$ is a cyclic representation of $X$ with respect to $T$, the sequence $\left(x_{n}\right)$ has infinite terms in each $A_{i}$ for $i \in\{1,2, \ldots, m\}$. Suppose that $x \in A_{i}, T x \in$ $A_{i+1}$ and we take a subsequence $x_{n_{k}}$ of $\left(x_{n}\right)$ with $x_{n_{k}} \in A_{i-1}$ (the existence of this subsequence is guaranteed by the previously mentioned comment). By using the contractive condition, we can obtain

$$
\begin{aligned}
d\left(x_{n_{k+1}}, T x\right)= & d\left(T x_{n_{k}}, T x\right) \\
\leq & \alpha\left[d\left(x_{n_{k}}, T x_{n_{k}}\right)+d(x, T x)+d\left(x_{n_{k}}, T x\right)+d\left(x, T x_{n_{k}}\right)\right) \\
& -\psi\left(d\left(x_{n_{k}}, T x_{n_{k}}\right), d(x, T x), d\left(x_{n_{k}}, T x\right), d\left(x, T x_{n_{k}}\right)\right) \\
= & \alpha\left[d\left(x_{n_{k}}, x_{n_{k+1}}\right)+d(x, T x)+d\left(x_{n_{k}}, T x\right)+d\left(x, x_{n_{k}+1}\right)\right) \\
& -\psi\left(d\left(x_{n_{k}}, x_{n_{k+1}}\right), d(x, T x), d\left(x_{n_{k}}, T x\right), d\left(x, x_{n_{k}+1}\right)\right) .
\end{aligned}
$$


Passing to the limit as $n \rightarrow \infty$ and using $x_{n_{k}} \rightarrow x$, lower semicontinuity of $\psi$, we have

$$
\begin{aligned}
d(x, T x) & \leq \frac{1}{2} d(x, T x)-\liminf _{n \rightarrow+\infty} \psi\left(d\left(x_{n_{k}}, x_{n_{k+1}}\right), d(x, T x), d\left(x_{n_{k}}, T x\right), d\left(x, x_{n_{k}+1}\right)\right) \\
& \leq \frac{1}{2} d(x, T x)-\psi(0, d(x, T x), d(x, T x), 0) \leq \frac{1}{2} d(x, T x)
\end{aligned}
$$

which is a contradiction unless $d(x, T x)=0$, and, therefore, $x$ is a fixed point of $T$. Finally, to prove the uniqueness of the fixed point, we have $y, z \in X$ with $y$ and $z$ being fixed points of $T$. The cyclic character of $T$ and the fact that $y, z \in X$ are fixed points of $T$ imply that $y, z \in \bigcap_{i=1}^{m} A_{i}$. Using the contractive condition we obtain

$$
\begin{aligned}
d(y, z)= & d(T y, T z) \leq \alpha[d(y, T y)+d(z, T z)+d(y, T z)+d(z, T y)] \\
& -\psi(d(y, T y), d(z, T z), d(y, T z), d(z, T y))
\end{aligned}
$$

that is,

$$
d(y, z) \leq 2 \alpha d(y, z)-\psi(0,0, d(y, z), d(z, y)) \leq d(y, z) .
$$

This gives us $\psi(0,0, d(y, z), d(z, y)) \leq(1-2 \alpha) d(y, z) \leq 0$, and, by our assumption about $\psi$, $d(y, z)=0$, that is, $y=z$. This finishes the proof.

Corollary 2.10. Let $(X, d)$ be a complete metric space, $m \in \mathbb{N}, A_{1}, A_{2}, \ldots, A_{m}$ nonempty subsets of $X$, and $X=\bigcup_{i=1}^{m} A_{i}$. Suppose that $T: X \rightarrow X$ be an operator such that

(1) $X=\bigcup_{i=1}^{m} A_{i}$ is a cyclic representation of $X$ with respect to $T$;

(2) there exists $\beta \in[0,1 / 4)$ such that

$$
d(T x, T y) \leq \beta[d(x, T x)+d(y, T y)+d(x, T y)+d(y, T x)]
$$

for any $x \in A_{i}, y \in A_{i+1}, i=1,2, \ldots, m$, where $A_{m+1}=A_{1}$. Then, $T$ has a fixed point $z \in \bigcap_{i=1}^{n} A_{i}$.

Proof. Let $\beta \in[0,1 / 4)$. Here, it suffices to take the function $\psi:[0,+\infty)^{4} \rightarrow[0,+\infty)$ defined by $\psi(a, b, c, e)=(\alpha-\beta)(a+b+c+e)$. Obviously, $\psi$ satisfies that $\psi(a, b, c, e)=0$ if and only if $a=b=c=e=0$, and $\psi(x, y, z, t)=(\alpha-\beta)(x+y+z+t)=\psi(x+y+z+t, 0)$. Then, we can apply Theorems 2.9 .

The following corollary gives us a fixed point theorem with a contractive condition of integral type for cyclic contractions.

Corollary 2.11. Let $(X, d)$ be a complete metric space, $m \in \mathbb{N}, A_{1}, A_{2}, \ldots, A_{m}$ nonempty closed subsets of $X$ and $X=\bigcup_{i=1}^{m} A_{i}$. Suppose that $T: X \rightarrow X$ be an operator such that

(1) $X=\bigcup_{i=1}^{m} A_{i}$ is a cyclic representation of $X$ with respect to $T$; 
(2) there exists $\beta \in[0,1 / 4)$ such that

$$
\int_{0}^{d(T x, T y)} \rho(t) d t \leq \beta \int_{0}^{d(x, T x)+d(y, T y)+d(x, T y)+d(y, T x)} \rho(t) d t
$$

for any $x \in A_{i}, y \in A_{i+1}, i=1,2, \ldots, m$, where $A_{m+1}=A_{1}$ and $\rho:[0, \infty) \rightarrow[0, \infty)$ is a Lebesgueintegrable mapping satisfying $\int_{0}^{\varepsilon} \rho(t) d t$ for $\varepsilon>0$. Then $T$ has unique fixed point $z \in \bigcap_{i=1}^{m} A_{i}$.

Proof. It is easily proved that the function $\varphi:[0, \infty) \rightarrow[0, \infty)$ given by $\varphi(t)=\int_{0}^{t} \rho(s) d s$ satisfies that $\varphi \in \mathbf{F}_{1}$. Therefore, Corollary 2.10 is obtained from Theorem 2.9, taking as $\varphi$ the perviously defined function and as $\psi$ the function $\psi(x, y, z, t)=(1 / 4-\alpha)(x+y+z+t)=$ $\varphi(x+y+z+t, 0)$.

If in Corollary 2.11, we take $A_{i}=X$ for $i=1,2, \ldots, m$, we obtain the following result.

Corollary 2.12. Let $(X, d)$ be a complete metric space and let $T: X \rightarrow X$ be a mapping such that for any $x, y \in X$,

$$
\int_{0}^{d(T x, T y)} \rho(t) d t \leq \beta \int_{0}^{d(x, T x)+d(y, T y)+d(x, T y)+d(y, T x)} \rho(t) d t
$$

where $\rho:[0, \infty) \rightarrow[0, \infty)$ is a Lebesgue-integrable mapping satisfying $\int_{0}^{\varepsilon} \rho(t) d t$ for $\varepsilon>0$ and the constant $\beta \in[0,1 / 4)$. Then $T$ has unique fixed point.

If in Theorem 2.9 we put $A_{i}=X$ for $i=1,2, \ldots, m$, we have the generalized result of

Corollary 2.13. Let $(X, d)$ be a complete metric space and let $T: X \rightarrow X$ be a mapping such that for any $x, y \in X$,

$$
\begin{array}{r}
d(T x, T y) \leq \alpha[d(x, T x)+d(y, T y)+d(x, T y)+d(y, T x)] \\
-\psi(d(x, T x), d(y, T y), d(x, T y), d(y, T x)),
\end{array}
$$

where $\psi \in \mathbf{F}_{2}$ and $\alpha \in(0,1 / 4]$. Then $T$ has unique fixed point.

Example 2.14. Let $X=\mathbb{R}$ with the usual metric. Suppose $A_{1}=[0,1], A_{2}=[0,1 / 3], A_{3}=$ $[0,1 / 9], A_{4}=[0,1 / 27]$ and $Y=\bigcup_{i=1}^{4} A_{i}$. Define $T: Y \rightarrow Y$ such that $T x=x / 4$ for all $x \in Y$. It is clear that $\bigcup_{i=1}^{4} A_{i}$ is a cyclic representation of $Y$ with respect to $T$. Furthermore, if $\psi:[0,+\infty)^{4} \rightarrow[0,+\infty)$ is defined by $\psi(x, y, z, t)=(x+y+z+t) / 16$, then $\psi \in \mathbf{F}_{2}$. As in Example 2.7, it can be easily shown that $T$ is a generalized cyclic weakly Chatterjea type contraction $\alpha=1 / 4$. Therefore, all conditions of Theorem 2.9 are satisfied, and so $T$ has a fixed point (which is $z=0 \in \bigcap_{i=1}^{4} A_{i}$ ).

\section{References}

[1] H. K. Nashine, "New fixed point theorems for mappings satisfying generalized weakly contractive condition with weaker control functions," Annales Polonici Mathematici, vol. 104, pp. 109-119, 2012. 
[2] E. Karapınar, "Weak $\varphi$-contraction on partial metric spaces and existence of fixed points in partially ordered sets," Mathematica Aeterna, vol. 1, no. 4, pp. 237-244, 2011.

[3] S. K. Chatterjea, "Fixed-point theorems," Comptes Rendus de l'Académie Bulgare des Sciences, vol. 25, pp. 727-730, 1972.

[4] B. S. Choudhury, “Unique fixed point theorem for weak C-contractive mappings," Kathmandu University Journal of Science, Engineering and Technology, vol. 5, no. 1, pp. 6-13, 2009.

[5] W. A. Kirk, P. S. Srinivasan, and P. Veeramani, "Fixed points for mappings satisfying cyclical contractive conditions," Fixed Point Theory, vol. 4, no. 1, pp. 79-89, 2003.

[6] C.-M. Chen, "Fixed point theory for the cyclic weaker Meir-Keeler function in complete metric spaces," Fixed Point Theory and Applications, vol. 2012, article 17, 2012.

[7] L. Cirić, N. Cakić, M. Rajović, and J. S. Ume, "Monotone generalized nonlinear contractions in partially ordered metric spaces," Fixed Point Theory and Applications, Article ID 131294, 11 pages, 2008.

[8] M. Derafshpour, S. Rezapour, and N. Shahzad, "On the existence of best proximity points of cyclic contractions," Advances in Dynamical Systems and Applications, vol. 6, no. 1, pp. 33-40, 2011.

[9] J. Jachymski, "Equivalent conditions for generalized contractions on (ordered) metric spaces," Nonlinear Analysis. Theory, Methods and Applications A, vol. 74, no. 3, pp. 768-774, 2011.

[10] E. Karapinar, "Fixed point theory for cyclic weak $\phi$-contraction," Applied Mathematics Letters, vol. 24, no. 6, pp. 822-825, 2011.

[11] E. Karapınar, I. M. Erhan, and A. Y. Ulus, "Fixed point theorem for cyclic maps on partial metric spaces," Applied Mathematics and Information Sciences, vol. 6, no. 1, pp. 239-244, 2012.

[12] E. Karapinar and K. Sadarangani, "Corrigendum to "fixed point theory for cyclic weak $\phi$-contraction" [Appl. Math. Lett. 24 (6) (2011) 822-825]," vol. 25, no. 10, pp. 1582-1584, 2012.

[13] E. Karapınar, "Fixed point theory for cyclic $(\phi-\varphi)$-contractions," Applied Mathematics Letters, vol. 24, no. 6, pp. 822-825, 2011.

[14] S. Karpagam and S. Agrawal, "Best proximity point theorems for cyclic orbital MeirKeeler contraction maps," Nonlinear Analysis, Theory, Methods and Applications, vol. 74, no. 4, pp. 1040-1046, 2011.

[15] G. S. R. Kosuru and P. Veeramani, "Cyclic contractions and best proximity pair theorems," 2011, http://128.84.158.119/abs/1012.1434v2.

[16] K. Neammanee and A. Kaewkhao, "Fixed points and best proximity points for multi-valued mapping satisfying cyclical condition," International Journal of Mathematical Sciences and Applications, vol. 1, no. 1, atricle 9, 2011.

[17] M. Păcurar and I. A. Rus, "Fixed point theory for cyclic $\varphi$-contractions," Nonlinear Analysis. Theory, Methods and Applications A, vol. 72, no. 3-4, pp. 2683-2693, 2010.

[18] M. A. Petric, "Best proximity point theorems for weak cyclic Kannan contractions," Filomat, vol. 25, no. 145, 154 pages, 2011.

[19] I. A. Rus, "Cyclic representations and fixed points," Annals of the Tiberiu Popoviciu Seminar of Functional Equations, Approximation and Convexity, vol. 3, pp. 171-178, 2005.

[20] S. Banach, "Sur les operations dans les ensembles abstraits et leur application aux equations integerales," Fundamenta Mathematicae, vol. 3, pp. 133-181, 1922. 


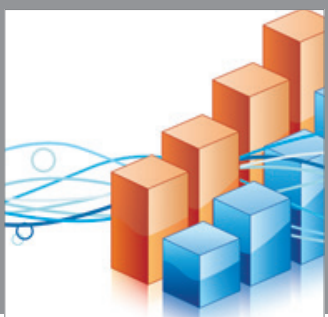

Advances in

Operations Research

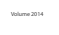

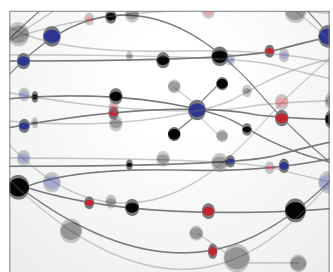

\section{The Scientific} World Journal
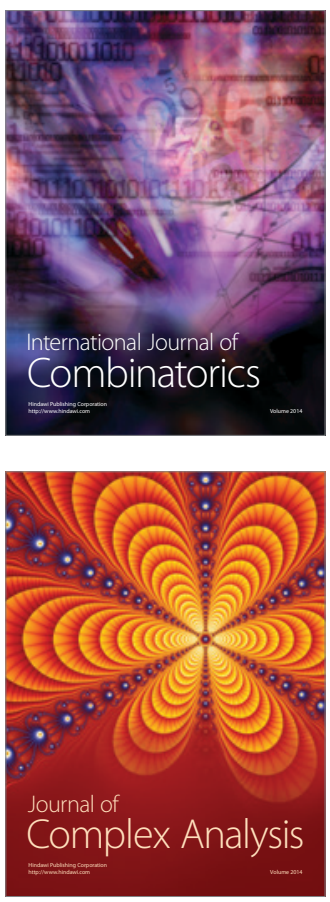

International Journal of

Mathematics and

Mathematical

Sciences
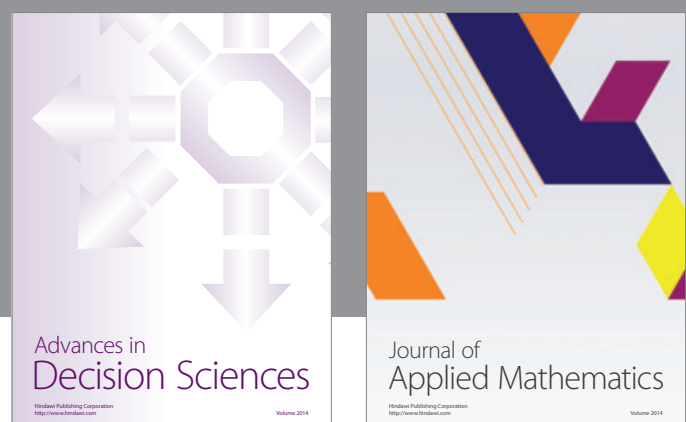

Journal of

Applied Mathematics
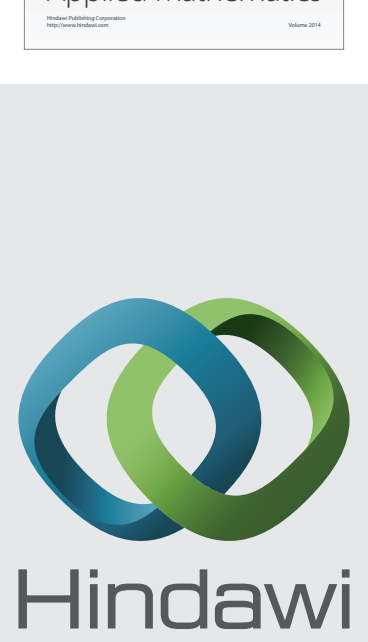

Submit your manuscripts at http://www.hindawi.com
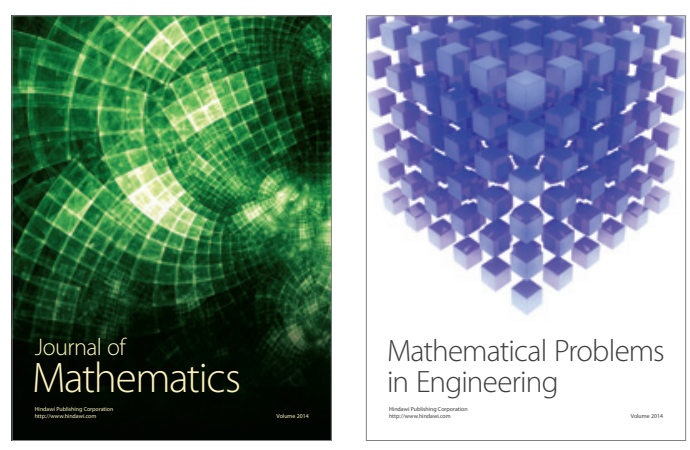

Mathematical Problems in Engineering
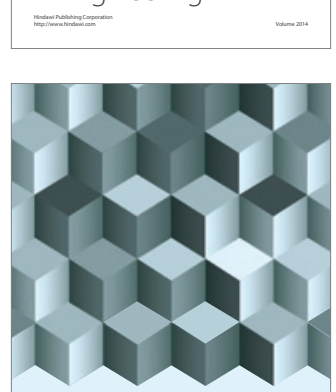

Journal of

Function Spaces
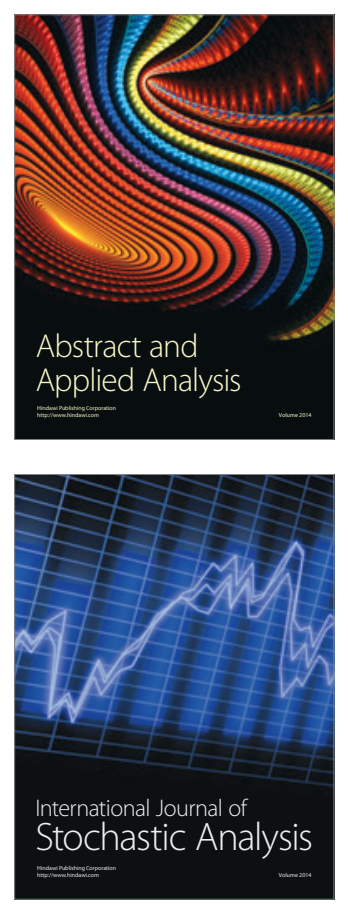

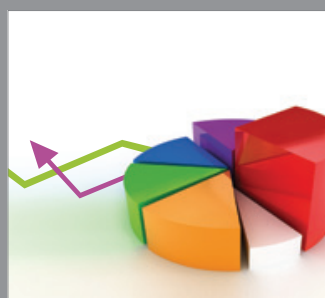

ournal of

Probability and Statistics

Promensencen
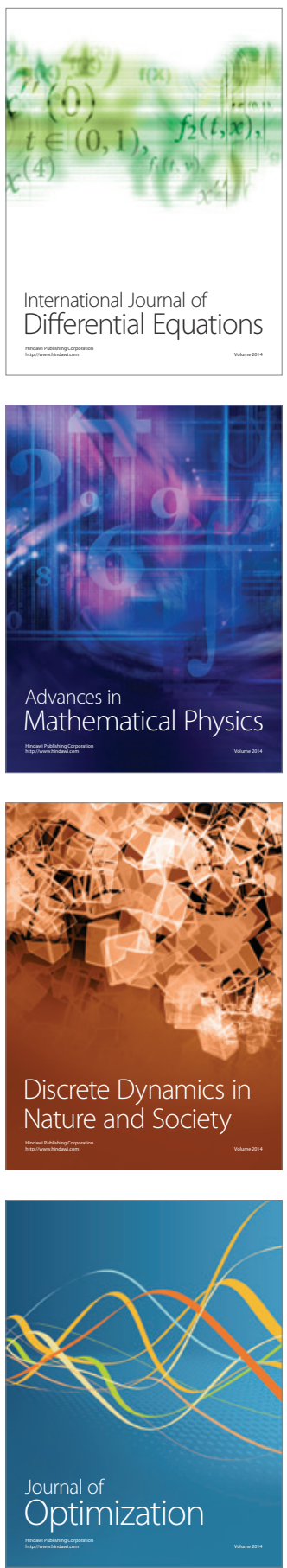